\title{
Selection of women at risk for cervical cancer in an HIV-infected South African population
}

\author{
Marjolein Van Zummeren, Wieke W. Kremer ${ }^{\mathrm{a}}$, Matthys C. Van Aardt ${ }^{\mathrm{b}}$, Erika Breytenbach ${ }^{\mathrm{b}}$, \\ Karin L. Richterc, Lawrence Rozendaal ${ }^{\mathrm{a}}$, Birgit I. Witte ${ }^{\mathrm{d}}$, Lise M.A. De Strooper ${ }^{\mathrm{a}}$, \\ Albertus T. Hesselinke, Danielle A.M. Heideman ${ }^{\mathrm{a}}$, Peter J.F. Snijders' ${ }^{\mathrm{a}}$, Renske D.M. Steenbergen ${ }^{\mathrm{a}}$, \\ Greta Dreyer ${ }^{\mathrm{b}}$ and Chris J.L.M. Meijer ${ }^{\mathrm{a},}{ }^{,}$
}

\begin{abstract}
${ }^{a}$ Cancer Centre Amsterdam, Department of Pathology, VU University Medical Centre, Amsterdam, The Netherlands, ${ }^{b}$ Department of Obstetrics and Gynaecology, Steve Biko Academic Hospital,

'Department of Medical Virology, University of Pretoria, and National Health Laboratory Service, Pretoria, South

Africa, ${ }^{\mathrm{d} D e p a r t m e n t}$ of Epidemiology and Biostatistics, VU University Medical Centre, and

eSelf-screen B.V., Amsterdam, The Netherlands.
\end{abstract}

${ }^{*}$ Correspondence to Chris J.L.M. Meijer, Cancer Centre Amsterdam, Department of Pathology, VU University Medical Centre, P.O. Box 7057, 1007 MB Amsterdam, The Netherlands.

Tel: +31 20444 4070; e-mail: cjlm.meijer@vumc.nl

Objective: Cervical cancer is the leading cause of cancer-related death in women in South Africa. This study evaluates DNA methylation levels in cervical (pre)cancer and aims to assess the value of high-risk human papillomavirus (hrHPV) testing and methylation analysis, alone or in combination, on physician-taken cervical scrapes to detect cervical cancer, and cervical intraepithelial neoplasia grade 3 (CIN3) in an HIV-infected South African population.

Design: Prospective observational multicentre cohort study.

Methods: Women from a cohort of women living with HIV $(n=355)$ and a referral cohort ( $n=109,60 \%$ HIV seropositive) were included. Cervical scrapes were collected for hrHPV testing and methylation analysis of cell adhesion molecule 1, T-lymphocyte maturation-associated protein, and microRNA124-2 genes. Histologic endpoints were available for all participants. Performance for detection of CIN3 or worse (CIN3+) was determined in the cohort of women living with HIV and different testing strategies were compared.

Results: HrHPV and methylation positivity rates increased with severity of cervical disease in the two study cohorts, each reaching $100 \%$ in samples of women with carcinoma. HrHPV testing showed a sensitivity for CIN3+ of $83.6 \%$, at a specificity of $67.7 \%$. Methylation analysis showed a comparable CIN3+ sensitivity of $85.2 \%$, but a significantly lower specificity of $49.6 \%$. HrHPV testing with reflex methylation analysis showed a CIN3 + sensitivity of $73.8 \%$, at a specificity of $81.5 \%$.

Conclusion: In this HIV-infected South African population, stratifying hrHPV-positive women with reflex methylation analysis detects all cervical carcinomas and yields an acceptable sensitivity and specificity for $\mathrm{CIN} 3+$.

Keywords: cytology, DNA methylation marker testing, early detection of cancer, high-grade cervical intraepithelial neoplasia, HIV, human papillomavirus DNA test, uterine cervical neoplasms 


\section{Introduction}

Cervical cancer remains an important public health problem in developing countries. Worldwide approximately 528000 women are diagnosed with cervical cancer and 266000 women die of the disease each year [1]. The majority of all these cancer cases (85\%) are diagnosed in low and middle-income countries (LMIC). In South Africa, cervical cancer is the leading cause of cancer-related death in women [2,3]. The lack of an effective cervical screening program and poor access to healthcare are the main contributors to this high mortality rate [4].

Cervical cancer is caused by a persistent infection with a high-risk type of the human papillomavirus (HPV) and develops through precancerous stages referred to as cervical intraepithelial neoplasia grade 1-3 (CIN1-3) [5-7]. An important risk factor for a persistent infection with HPV and the development of CIN and cervical cancer is infection with HIV [8,9]. In South Africa, approximately seven million people are infected with HIV (accounting for an estimated 12.7\% of the population), with women more severely affected than men [10]. This large high-risk population highlights the need of an effective cervical cancer prevention strategy.

Current cytology-based screening programs have proven difficult to implement in LMIC because of inadequate infrastructure and limited resources. South Africa has a national policy for cervical screening using cytology, but coverage of the program is limited to $16.7 \%$ [4]. Furthermore, the sensitivity of cytology for detection of cervical disease has a wide variability, likely attributable to the subjective nature of the test [11]. An ideal cervical screening strategy should be objective and robust leading to effective cervical (pre)cancer detection.

An alternative for cytology-based screening is HPV-based screening. Screening for high-risk HPV (hrHPV) leads to earlier detection of CIN3 and cervical cancer (CIN3+) when compared to cytology [12-15], and provides a better protection against cervical cancer [16-21]. For this reason, hrHPV testing will replace cytology as a primary screening test in several countries [22]. However, the specificity of hrHPV testing is limited as many of the detected infections are transient, and only a minority is associated with cervical abnormalities $[23,24]$. Therefore, subsequent testing of hrHPV-positive women is recommended to identify women with clinically meaningful disease and to prevent overreferral and overtreatment $[25,26]$.

An attractive molecular tool for detection of CIN3+ in hrHPV-positive women is the analysis of host cell DNA methylation of genes related to cervical cancer development [27-30]. Hypermethylation of the promoter regions of the tumour suppressor genes cell adhesion molecule 1 (CADM1), T-lymphocyte maturation-associated protein $(M A L)$, and microRNA-124-2 (miR1242), leads to gene silencing and is recognized as an essential step in cervical carcinogenesis [31-35]. Methylation levels of these genes increase with the severity and duration of the underlying cervical disease and are exceptionally high in cervical cancer [36,37]. We previously showed that DNA methylation analysis of cervical scrapes for CADM1, MAL, and miR124-2 by a clinically validated multiplex quantitative methylationspecific polymerase chain reaction (PCR) detected all cervical cancers [37]. Furthermore, methylation levels of these genes are significantly higher in CIN2/3 with a long-standing ( $\geq 5$ years) persistent hrHPV infection (so called advanced CIN2/3 lesions), compared with CIN2/ 3 associated with a more recently acquired infection ( $<5$ years) [36]. Accordingly, methylation positivity is claimed to be associated with advanced CIN2/3 with a high short-term progression risk to cervical cancer, in need of direct treatment [27]. This high sensitivity for advanced CIN2/3 and particularly cervical cancer makes these markers attractive screening tools in countries with limited screening lifetime opportunities [38].

The aim of the present study was to assess the clinical performance of hrHPV testing and methylation analysis, as single or combined testing strategies, for the detection of cervical cancer and CIN3+ in an HIV-infected population in South Africa.

\section{Methods}

\section{Study participants and procedures}

Between February 2013 and March 2015, women aged 18 years or above were recruited for participation in a prospective observational multicentre cohort study among women visiting a gynaecological outpatient clinic at either the Steve Biko Academic Hospital or the Tshwane District Hospital, in Pretoria, South Africa. The study protocol was approved by the Ethical Committee of the University of Pretoria, South Africa (protocol numbers 100/2012 and 155/2014). All participants gave written informed consent. The study comprised a cohort of women living with HIV (WLHIV) and a referral cohort (Fig. 1).

\section{South African cohort of women living with HIV}

In the cohort of WLHIV, women who tested HIV seropositive and visited the gynaecological outpatient clinic for cervical screening by a cervical scrape were included. All women with a recent history of cervical pathology (treated CIN2+ within two years prior to inclusion) were excluded from the study, as were women who previously underwent a hysterectomy. After obtaining informed consent, a questionnaire on women's characteristics including the use of antiretroviral therapy 


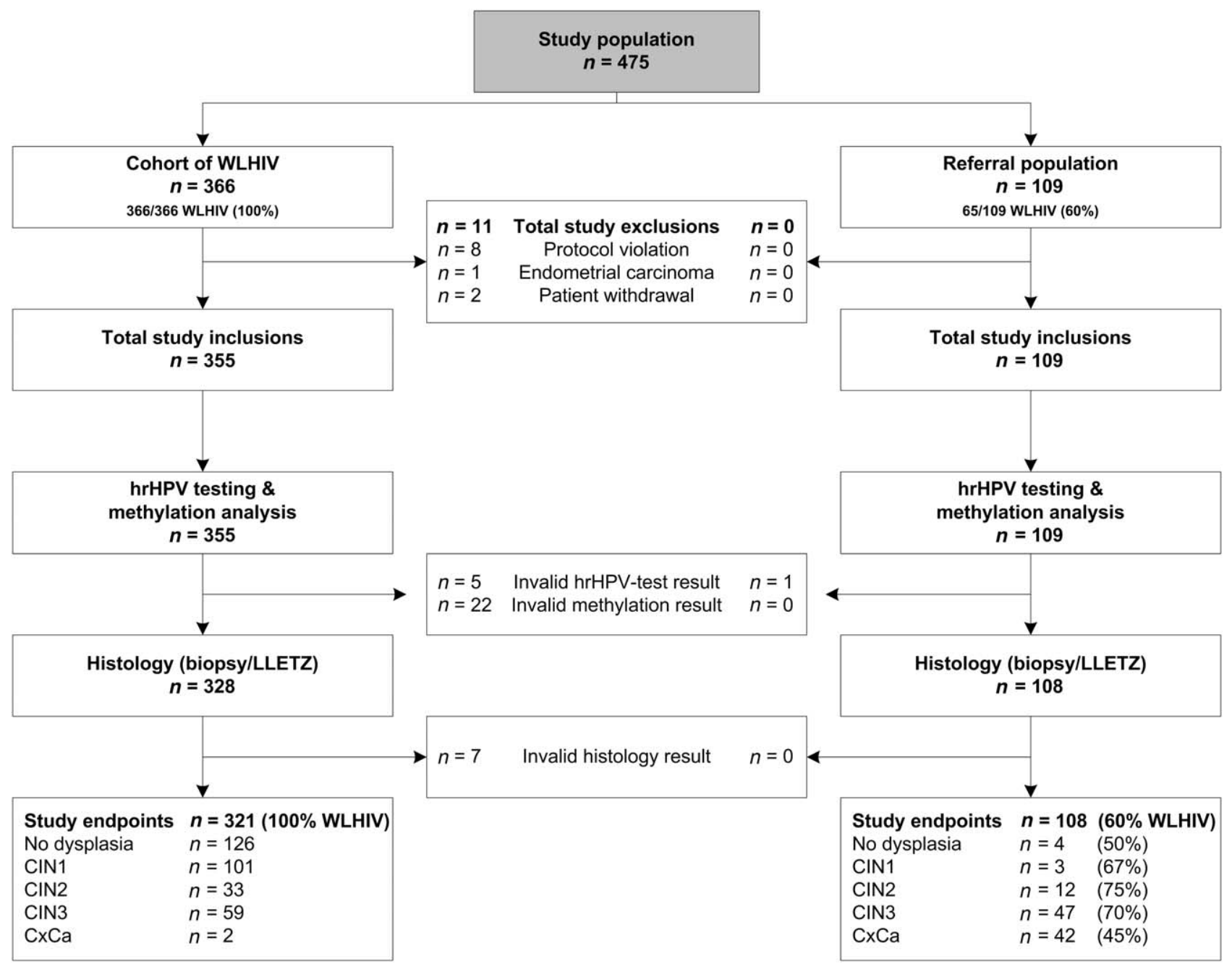

Fig. 1. Study flowchart. CIN, cervical intraepithelial neoplasia; $\mathrm{CxCa}$, cervical carcinomas; hrHPV, high-risk human papillomavirus; LLETZ, large loop excision of the transformation zone; WLHIV, women living with HIV.

and $\mathrm{CD}^{+}{ }^{+}$cell count was administered, cervical cells were collected using a Cervix-Brush (Rovers Medical Devices B.V., Oss, the Netherlands) and in all women colposcopy was performed by a trained physician. After preparation of a conventional Pap smear, the remaining cervical scrape material was stored in $20 \mathrm{ml}$ of Thinprep PreservCyt solution (Hologic, Marlborough, Massachusetts, USA). At colposcopy, two mandatory cervical biopsies were taken either from the most abnormal area on the cervix, or at random (6 and 12 o'clock) if no lesion was visible. Endocervical curettage was performed if the squamocolumnar junction could not be visualized.

\section{South African referral population}

To investigate hrHPV and methylation positivity in different histology groups, a referral cohort was included in the study protocol to enrich the group of CIN2/3 and particularly cervical carcinoma. In this referral cohort, further referred to as 'referral population', women who visited the gynaecologic outpatient department for evaluation of abnormal cytology [high-grade squamous intraepithelial lesion or worse ( $\geq$ HSIL)] or biopsy-proven cervical cancer were included. Participants in this study cohort were also subjected to a clinical questionnaire and a cervical scrape was taken using a Cervix-Brush. This cervical scrape material was stored in $20 \mathrm{ml}$ of Thinprep PreservCyt solution (Hologic). Participants were clinically managed according to their referral reason (see Study endpoints).

\section{Dutch reference population}

Because the number of HIV seronegative women in the two South African cohorts was too low to assess methylation differences between HIV-infected and non-HIV-infected women (see Statistical analysis), we enlarged the sample size of HIV seronegative women for this specific analysis with a Dutch hrHPV-positive reference population $[n=188$; study endpoints: 77 $\leq \mathrm{CIN} 1,32$ CIN2, 16 CIN3, and 63 cervical carcinomas; mean age: 41 years (range: $18-85$ )] [39]. As the incidence of HIV in the Netherlands is very low, the Dutch study group was assumed to be HIV seronegative [40]. This 
cohort was not included in the original study protocol and only used for this analysis.

\section{Study endpoints}

Conventional cytology slides and liquid-based cytology (LBC) preparations were assessed through regular cervical screening procedures and classified according to the Bethesda 2001 classification [41]. All biopsies were classified as no dysplasia, CIN1, CIN2, CIN3, or invasive cancer, according to international criteria [42].

Women with atypical squamous cells - cannot exclude HSIL (ASC-H) or HSIL on cytology (conventional or LBC) or CIN2+ on a cervical biopsy were treated with a large loop excision of the transformation zone (LLETZ), according to local guidelines. Gynaecological examination for clinical staging was performed in women referred for cervical cancer. LLETZ specimens were also classified as no dysplasia, CIN1, CIN2, CIN3, or invasive cancer. No cervical adenocarcinoma in-situ lesions were found in the study population. Worst histology on either the cervical biopsy or LLETZ specimen was taken as study endpoint. If no diagnosis could be made based on biopsy or LLETZ specimens, a sample was considered invalid for histology.

\section{High-risk human papillomavirus testing}

Vials containing cellular material in PreservCyt medium were shipped to the Department of Pathology at the VU University Medical Centre, Amsterdam, the Netherlands. DNA was isolated from the cervical LBC material using the Nucleo-Spin 96 Tissue kit (Macherey-Nagel, Düren, Germany) and a Microlab Star robotic system (Hamilton, Germany) according to manufacturers' instructions [43]. $\beta$-Globin PCR analysis was conducted to confirm the presence of human DNA in all specimens. The presence of hrHPV was determined using the clinically validated $\mathrm{GP}^{+} / 6^{+}$PCR-EIA $[44,45]$. This kit detects DNA from HPV genotypes 16, 18, 31, 33, 35, 39, 45, 51, 52, 56, 58, 59, 66, and 68 (DDL, Rijswijk, the Netherlands). A hrHPV and $\beta$-globin negative sample was considered invalid.

\section{Methylation analysis}

Isolated DNA from the cervical scrapes was subjected to bisulfite treatment using the EZ DNA Methylation Kit (Zymo Research, Irvine, California, USA) as described previously [32,33]. A commercial, clinically validated multiplex quantitative methylation-specific PCR (PreCursor-M, Self-screen B.V., Amsterdam, the Netherlands) was used for DNA methylation analysis of CADM1, MAL, and miR124-2 [37]. $\beta$-actin was used as a methylation-independent reference to assure sample quality. Analyses were done on an ABI 7500 real-time PCR system (Applied Biosystems, Foster City, California, USA). A sample was scored invalid for methylation analysis when quantification cycle value for $\beta$-actin was more than 30. Methylation values of CADM1, MAL, and miR124-2 were normalized to the reference gene $\beta$-actin using the comparative quantification cycle method for each marker: $100 \times 2(\mathrm{Ct}(\beta$-actin $)-\mathrm{Ct}($ target $))$, resulting in cycle threshold $(\mathrm{Ct})$ ratios [46].

\section{Statistical analysis}

HrHPV positivity was determined in different histology subgroups for the cohort of WLHIV and the referral population separately.

To assess differences between methylation levels of CADM1, MAL, and miR124-2 in different histology groups, log-transformed $\mathrm{Ct}$ ratios of the different methylation markers were plotted in a boxplot per histology group (no dysplasia, CIN1, CIN 2, CIN3, and cervical carcinomas) for the cohort of WLHIV and the referral population together.

In addition, we analysed the effect of HIV status on CADM1, MAL, and miR124-2 levels in the different histology groups. Differences between HIV seropositive from the two South African cohorts and seronegative samples from the South African referral and the Dutch reference population per histology group were assessed by Mann-Whitney $U$ tests for the log-transformed Ct ratios of the methylation markers.

We then determined CADM1, MAL, and miR124-2 positivity in different histology groups for the cohort of WLHIV and the referral population. According to the manufacturers' instructions, a sample was considered methylation marker positive if the $\mathrm{Ct}$ ratio of at least one of the individual methylation markers CADM1, MAL, and miR124-2 was above the described clinically validated thresholds [39].

Finally, in the cohort of WLHIV, the sensitivity for the detection of CIN3+ as primary outcome measure by hrHPV testing, methylation analysis and hrHPV testing with reflex methylation analysis (that is, hrHPV-positive women were stratified by methylation analysis and considered positive if both assays tested positive among all women screened) was calculated. The sensitivity and specificity for CIN2+ are shown in supplementary data (Supplementary Table 1, http://links.lww.com/QAD/ B131). Sensitivities and specificities of the different testing strategies were compared using the McNemar test, and $95 \%$ confidence intervals $(\mathrm{CI})$ were computed for the relative sensitivities and specificities [47]. Calculations were performed in Microsoft Excel (2010), SPSS (V.22), and STATA (V14.1).

\section{Results}

\section{Baseline characteristics}

In total, 475 women were enrolled in this study, of whom 366 in the cohort of WLHIV and 109 in the referral 
Table 1. Performance of different screening methods.

\begin{tabular}{|c|c|c|c|c|c|c|}
\hline Screening method & $\mathrm{CIN} 3+$ sensitivity $^{\mathrm{a}}$ & $95 \% \mathrm{Cl}$ & $n 1 / N 1$ & CIN3+ specificity & $95 \% \mathrm{Cl}$ & $n 2 / N 2$ \\
\hline \multicolumn{7}{|l|}{ Cohort of WLHIV $(n=321)$} \\
\hline HrHPV testing & $83.6 \%$ & $74.3-92.9$ & $51 / 61$ & $67.7 \%$ & $62.0-73.4$ & $176 / 260$ \\
\hline Methylation analysis & $85.2 \%$ & $76.3-94.1$ & $52 / 61$ & $49.6 \%$ & $43.5-55.7$ & $129 / 260$ \\
\hline HrHPV testing with reflex methylation analysis & $73.8 \%$ & $62.7-84.8$ & $45 / 61$ & $81.5 \%$ & $76.8-86.3$ & $212 / 260$ \\
\hline
\end{tabular}

Test specifications of high risk human papillomavirus testing, methylation analysis, and combined high-risk human papillomavirus testing with reflex methylation analysis for the detection of cervical intraepithelial neoplasia 3 or worse $(\mathrm{CIN} 3+)$. $\mathrm{Cl}$, confidence interval; $\mathrm{CIN}$, cervical intraepithelial neoplasia; HrHPV, high risk human papillomavirus; n1, number of test positive disease cases; N1, total number of disease cases; n2, number of test negative nondisease cases; N2, total number of nondisease cases.

${ }^{\mathrm{a}}$ No carcinomas were missed by any of the presented screening strategies.

population (Fig. 1). In the cohort of WLHIV, 11 women were excluded because of protocol violation $(n=8)$, presence of an endometrial carcinoma $(n=1)$, and patient withdrawal $(n=2)$, leaving 355 women. In the referral population, no women were excluded.

The median age in the cohort of WLHIV was 40 years [interquartile range (IQR): 35-46 years] and 44 years (IQR: 34-51 years) in the referral population. In the cohort of WLHIV all women were HIV seropositive, of whom $353(99 \%)$ were on antiretroviral treatment. The median $\mathrm{CD}^{+}$cell count at time of cervical screening in the cohort of WLHIV was 514 cells/ $\mu$ l (IQR: $380-720$ cells $/ \mu \mathrm{l}$ ). In the referral population, 65 women $(60 \%)$ were HIV seropositive, of whom 24 (37\%) were on antiretroviral treatment. Median $\mathrm{CD}^{+}$cell count in this population was 342 cells/ $\mu$ l (IQR: 236506 cells $/ \mu \mathrm{l})$. In total 38 women $(35 \%)$ were HIV seronegative and five (5\%) women had an unknown HIV status.

Women with an invalid HPV test result, invalid methylation test result, or without a histologic endpoint were excluded from analyses. In the cohort of WLHIV, this resulted in 321 women with valid test results and a study endpoint: 126 women had no dysplasia, 101 women had CIN1, 33 women had CIN2, 59 women had CIN3, and two women had cervical carcinoma [one squamous cell carcinoma (SCC) and one adenocarcino$\mathrm{ma}$.

In the referral population, 108 women had valid test results and a study endpoint. Of $65 \mathrm{HIV}$ seropositive women in this cohort, two women had no dysplasia, two women had CIN1, nine women had CIN2, 33 women had CIN3, and 19 women had cervical carcinoma (17 SCC, one adenocarcinoma, and one not specified). Of the 38 HIV seronegative women in this cohort, two women had no dysplasia, two women had CIN2, 12 women had CIN3, and 22 women had cervical carcinoma (19 SCC and three not specified). Of the five women with unknown HIV status in this cohort, one woman had CIN1, one woman had CIN2, two women had CIN3, and one woman had cervical carcinoma type SCC.

\section{High-risk human papillomavirus testing}

High-risk human papillomavirus positivity rate

In the cohort of WLHIV, in total 135 women (42\%) tested positive for hrHPV. Positivity rate increased with the severity of the underlying cervical disease: hrHPV positivity was found in $30(24 \%)$ women without dysplasia, 31 (31\%) women with CIN1, 23 (70\%) women with CIN2, $49(83 \%)$ women with CIN3, and two $(100 \%)$ women with cervical carcinoma.

In the referral population, in total 103 women (95\%) tested positive for hrHPV. HrHPV positivity in HIV seropositive women was found in two $(100 \%)$ women without dysplasia, eight $(89 \%)$ women with CIN2, 33 $(100 \%)$ women with CIN3, and 19 (100\%) women with cervical carcinoma. HrHPV positivity in HIV seronegative women was found in one $(50 \%)$ woman without dysplasia, two (100\%) women with CIN2, 11 (92\%) women with CIN3, and $22(100 \%)$ women with cervical carcinoma. All women with unknown HIV status $(n=5$, one CIN1, one CIN2, two CIN3, one SCC) tested positive for hrHPV.

\section{Methylation analysis}

Methylation marker levels

As shown in Fig. 2, in the South African study cohorts methylation levels of CADM1 (Fig. 2a), MAL (Fig. 2b), and miR124-2 (Fig. 2c) increased with the severity of the underlying cervical disease.

To investigate a potential effect of HIV status on methylation levels of the three markers, we compared Ct ratios of CADM1, MAL, and miR124-2 between HIV seropositive $(n=386$, South African population) and HIV seronegative $(n=226$; South African population $n=38$; Dutch population $n=188$ ) women. South African cases with unknown HIV status were excluded from this analysis $(n=5)$. Ct ratios of $m i R 124-2$ were significantly increased in the samples of HIV seropositive women compared with those of HIV seronegative women with CIN1 $(P<0.001)$ or less, CIN2 $(P=0.003)$, and CIN3 $(P=0.031)$. This effect was not seen among samples of women with cancer $(P=0.46)$. The Ct ratios for CADM1 and MAL were not 
(a)

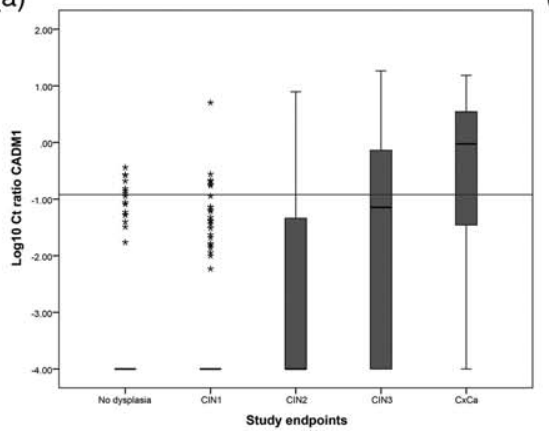

(b)

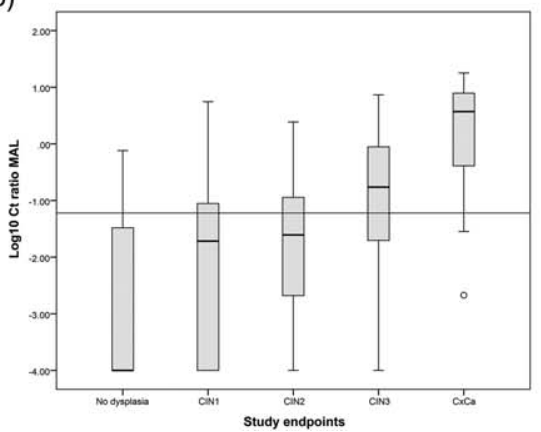

(c)

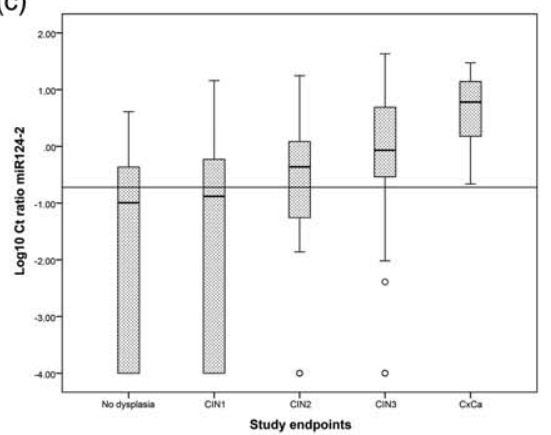

Fig. 2. Methylation levels increase with severity of histology study endpoints. Methylation levels of CADM1 (a), MAL (b) and miR124-2 (c) in cervical scrapes represented by boxplots of the log10-transformed Ct ratios (y-axis) in the different histology study endpoint groups (x-axis) from the cohort of women living with HIV and referral cohort combined. The threshold for marker positivity is given as a reference line. CIN, cervical intraepithelial neoplasia; CxCa, cervical carcinomas.

significantly increased in HIV seropositive women over HIV seronegative women.

Similar findings were observed when comparing South African HIV seropositive samples $(n=386)$ with Dutch HIV seronegative samples $(n=188)$.

\section{Methylation positivity rate}

In the cohort of WLHIV, in total 183 women (57\%) tested positive for methylation of one or more markers. The methylation positivity rate increased with the severity of the underlying cervical disease: that is, methylation positivity was detected in 55 (44\%) women without dysplasia, 55 (54\%) women with CIN1, 21 (64\%) women with CIN2, 50 (85\%) women with CIN3, and two $(100 \%)$ women with cervical carcinomas.

In the referral population, in total 97 women (90\%) tested positive for methylation of one or more markers. Methylation positivity in HIV seropositive women was detected in one $(50 \%)$ woman without dysplasia, one (50\%) woman with CIN1, seven (78\%) women with CIN2, 32 (97\%) women with CIN3, and 19 (100\%) women with cervical carcinomas. Methylation positivity in HIV seronegative women was detected in two (100\%) women with CIN2, nine (75\%) women with CIN3, and $22(100 \%)$ women with cervical carcinomas. All women with unknown HIV status $(n=5$, one CIN1, one CIN2, two CIN3, one SCC), except the woman with CIN2, tested positive for methylation of one or more markers.

\section{Performance of testing strategies}

Test specifications for the detection of CIN3+ of hrHPV testing, methylation analysis, and hrHPV testing with reflex methylation analysis in WLHIV are shown in Table 1.

HrHPV testing scored all cervical cancers positive and yielded a high sensitivity for the detection of CIN3+ of $83.6 \%$, with a specificity of $67.7 \%$. Methylation analysis also scored all cancers positive and showed a sensitivity for
CIN3 + of $85.2 \%$, comparable to hrHPV testing, but with a significantly lower specificity of $49.6 \%(P<0.001$; ratio 0.73 ; 95\%CI 0.64-0.85). HrHPV testing with reflex methylation analysis led to a significantly lower sensitivity for CIN3 + over sole hrHPV testing of 73.8\% $(P=0.031$; ratio 0.88 ; 95\%CI $0.80-0.98)$, but with a significant increase in specificity over sole hrHPV testing, reaching $81.5 \% \quad(P<0.001 ;$ ratio $1.21 ; 95 \%$ CI $1.13-1.28)$. Comparable results were found for the detection of CIN2+ (Supplementary Table 1, http://links.lww.com/ QAD/B131).

In the referral population test performances in terms of sensitivity and specificity for CIN3+ could not be calculated because the selection of women was only based on abnormal cytology.

\section{Discussion}

In this prospective observational multicentre cohort study in an HIV-infected South African population, both hrHPV testing and methylation analysis of the genes CAMD1, MAL, and miR124-2 (PreCursor-M) detected all cervical carcinomas and the majority of CIN3, with a moderate CIN3+ specificity for both hrHPV testing (67.7\%) and methylation analysis (49.6\%). This specificity was significantly increased $(81.5 \%)$ by the use of methylation analysis as a reflex test among hrHPVpositive women, with an acceptable sensitivity (73.8\%). The combination of these two molecular tests offers an objective screening strategy which provides a high reassurance against cervical cancer.

The specificity of methylation analysis, as performed in this study using the marker panel of CADM1, MAL, and miR124-2, would need improvement when considered as primary screening method in this population living with HIV. The relatively low specificity could be explained by the significantly higher methylation levels 
for miR124-2 found in control samples (CIN1 or less) of HIV seropositive women when compared with control samples of HIV seronegative women. The relatively higher methylation levels might be explained by the upregulation of DNA methyltransferase expression and activity, resulting in increased DNA methylation levels in HIV-infected cells [48,49]. A methylation panel fine-tuned specifically for testing populations with a high HIV prevalence, potentially including different markers, might correct for this and warrants further investigation [28,50,51]. As we had to include a Dutch seronegative population to reach sufficient power for comparative analyses, we cannot exclude that the difference between HIV seropositive and seronegative women may in part reflect a population effect. The relatively low specificity for CIN3 + detection by hrHPV testing in the WLHIV cohort might be explained by the high prevalence of hrHPV in this population and/or an effect of immunosuppression caused by an HIV infection $[52,53]$.

Methylation analysis as a stand-alone test for the detection of cervical abnormalities may provide an interesting screening method in LMIC with poor access to healthcare and low lifetime screening opportunities, where the optimal screening tool should be cancer proof and preferably applicable in one visit to prevent loss to follow-up. In this study, methylation analysis had a comparable sensitivity as hrHPV testing, and identified all cervical carcinomas and the majority of CIN3 in both the cohort of WLHIV and the referral population. Our findings are in line with previous research $[38,54,55]$. De Vuyst et al. evaluated the same methylation marker panel of CADM1, MAL, and miR124-2 for the stratification of hrHPV-positive women in a cross-sectional study of HIV seropositive women in Kenya [38]. Triage by this marker set was clearly superior to HPV16/18 genotyping or visual inspection with acetic acid and comparable to cytology. We found comparable results for HPV16/18 genotyping triage in this study (M.V.Z. and W.W.K. manuscript in preparation). In a case control study in Tanzania with a high HIV prevalence, other methylation markers [paternally expressed gene 3 (PEG3), insulin-like growth factor 2 (IGF2), H19 differentially methylated region (H19 DMR) and paternally expressed gene $1 /$ mesoderm-specific transcript (PEG1/MEST)] were also found to be associated with an increased risk of CIN and cervical cancer $[54,55]$.

Cytology was not shown as a reference strategy because of the bias in the performance of cytology compared to hrHPV testing and methylation analysis. The indication for treatment was only based either on HSIL on cytology, or CIN2+ on biopsy. Therefore, performance of cytology could not directly be compared to hrHPV testing and methylation analysis $[51,56]$. Nonetheless, we calculated the performance of cytology ( $\geq$ HSIL); the sensitivity for CIN3+ was $57.4 \%$ with a specificity of
92.6\%. The sensitivity for CIN2+ was $48.9 \%$ with a specificity of $96.4 \%$.

A limitation of the study is that hrHPV testing and methylation analysis were performed in an expert laboratory in the Netherlands. Therefore, the performance of the assays might not truly represent field conditions. Moreover, it cannot be excluded that the shipment of the samples influenced the quality of the samples, possibly explaining the number of test failures for hrHPV testing of $1.3 \%$ and methylation analysis of $4.7 \%$. In the future, test performance evaluations should be done locally.

An important advantage of cervical screening by hrHPV testing and methylation analysis is the objective nature of the tests. By using these tests, cervical screening could become independent of (subjective) cytology testing. Furthermore, their applicability to self-collected cervical material will be an interesting addition in the optimization of screening programs, as shown in previous trials [57-59]. These molecular tests can be further improved into rapid point-of-care tests that allow women to be (self)screened and, if local facilities allow, treated in one day. This would restrict the loss to follow-up and decrease the workload for gynaecologists. However, local infrastructure and cost-effectiveness in cervical screening are important aspects in LMIC [38]. If molecular tests are used more widely, the emergence of affordable tests can be envisioned and implementation in LMIC would become more feasible.

The study shows a promising clinical performance of hrHPV testing and methylation analysis for the detection of CIN3+ in an HIV-infected population in South Africa. Under current settings, hrHPV testing with reflex methylation analysis of CADM1, MAL, and miR124-2 in hrHPV-positive women could be an alternative objective, biomarker-based test strategy. It allows the detection of all cervical carcinomas and yields a high sensitivity with a good specificity for CIN3+. In future, primary methylation analysis could be an attractive screening tool in LMIC if specificity can be increased. Local settings such as available resources and population characteristics should determine which strategy is most favourable.

\section{Acknowledgements}

We gratefully acknowledge all women participating in this study trial. Furthermore, we are grateful for the active cooperation of the teams of the clinics of the department of Obstetrics and Gynaecology of the Steve Biko Academic Hospital and the HIV clinic at the Tshwane District Hospital. We would like to thank all the research staff and (cyto-) technicians of the Department of Pathology VUmc. 
G.D. and C.J.L.M.M. are principal investigators of the study. M.Z., W.W.K., M.C.A., E.B., P.J.F., G.D., and C.J.L.M. have set up the trial. M.Z., W.W.K., M.C.A., E.B., K.L.R., L.R., L.M.A.D.S., A.T.H., D.A.M.H., P.J.F.S., R.D.M.S., G.D., and C.J.L.M.M. were involved in data collection. M.Z., W.W.K., and B.I.W. performed the statistical analysis. M.Z. and W.W.K. managed the database. M.Z., W.W.K., R.D.M.S., and C.J.L.M.M. drafted the manuscript. All authors critically reviewed the manuscript and approved the final version. All authors had full access to all of the data in the study and can take responsibility for the integrity of the data and the accuracy of the data analysis and believe that the manuscript represents honest work. C.J.L.M.M. affirms that the manuscript is an honest, accurate, and transparent account of the study being reported; that no important aspects of the study have been omitted; and that any discrepancies from the study as planned have been explained.

The study was funded by the VU University Research Fellowship (URF) program (Amsterdam, the Netherlands), the 1st For Women Foundation (Pretoria, South Africa), and the Carl \& Emily Fuchs Foundation (Pretoria, South Africa).

\section{Conflicts of interest}

K.L.R. has received speakers fee from Roche diagnostics and MSD; A.T.H. is employee of Self-screen B.V.; D.A.M.H., P.J.F.S., R.D.M.S., and C.J.L.M.M. are minority shareholders of Self-screen B.V., a spin-off company of VUmc; Self-screen B.V. holds patents related to the work (i.e. hrHPV test and methylation markers for cervical screening); D.A.M.H. serves occasionally on the scientific advisory boards of AMGEN and Pfizer; P.J.F.S. has been on the speakers bureau of Roche diagnostics, Gen-Probe, Abbott, Qiagen, and Seegene and has been a consultant for Crucell B.V.; C.J.L.M.M. has received speakers fee from GSK, Qiagen, SPMSD/Merck, Roche diagnostics, Menarini, and Seegene, served occasionally on the scientific advisory board (expert meeting) of GSK, Qiagen, SPMSD/Merck, Roche, and Genticel and has been by occasion consultant for Qiagen and Genticel; C.J.L.M.M. was minority shareholder of Diassay B.V. until April 2016, until 2014 he held a small number of certificates of shares in Delphi Biosciences, which went into receivership in 2014.

Data presented previously at $31^{\text {st }}$ International Papillomavirus Conference, HPV 2017, South Africa, and published as abstract in the conference abstract book.

\section{References}

1. Ferlay J, Soerjomataram I, Dikshit R, Eser S, Mathers C, Rebelo $M$, et al. Cancer incidence and mortality worldwide: sources, methods and major patterns in GLOBOCAN 2012. Int / Cancer 2015; 136:E359-E386.
2. Bruni L, Barrionuevo-Rosas L, Albero G, Aldea M, Serrano B, Valencia $S$, et al. Human papillomavirus and related diseases report, South Africa. ICO information Centre on HPV and Cancer 2016.

3. Forman D, Brewster BF, Gombe Mbalawa DH, Kohler C, Pineros B, Steliarova-Foucher $M$, et al. Cancer Incidence in Five Continents. IARC Scientific Publications 2014X(164).

4. Adonis L, An R, Luiz J, Mehrotra A, Patel D, Basu D, Sturm R. Provincial screening rates for chronic diseases of lifestyle, cancers and HIV in a health-insured population. S Afr Med J 2013; 103:309-312.

5. Walboomers JM, Jacobs MV, Manos MM, Bosch FX, Kummer $\mathrm{JA}$, Shah KV, et al. Human papillomavirus is a necessary cause of invasive cervical cancer worldwide. J Pathol 1999; 189:12-19.

6. zur Hausen $\mathrm{H}$. Papillomaviruses and cancer: from basic studies to clinical application. Nat Rev Cancer 2002; 2:342-350.

7. Gius D, Funk MC, Chuang EY, Feng S, Huettner PC, Nguyen L, et al. Profiling microdissected epithelium and stroma to model genomic signatures for cervical carcinogenesis accommodating for covariates. Cancer Res 2007; 67:7113-7123.

8. Chaturvedi AK, Madeleine MM, Biggar RJ, Engels EA. Risk of human papillomavirus-associated cancers among persons with AIDS. J Natl Cancer Inst 2009; 101:1120-1130.

9. Denslow SA, Rositch AF, Firnhaber C, Ting J, Smith JS. Incidence and progression of cervical lesions in women with HIV: a systematic global review. Int J STD AIDS 2014; 25:163-177.

10. UNAIDS. Prevention GAP Report. 2016.

11. Kitchener HC, Castle PE, Cox JT. Chapter 7: Achievements and limitations of cervical cytology screening. Vaccine 2006; 24 (Suppl 3):S3/63-S3/70.

12. Bulkmans NW, Berkhof J, Rozendaal L, van Kemenade FJ, Boeke AJ, Bulk S, et al. Human papillomavirus DNA testing for the detection of cervical intraepithelial neoplasia grade 3 and cancer: 5-year follow-up of a randomised controlled implementation trial. Lancet 2007; 370:1764-1772.

13. Mayrand MH, Duarte-Franco E, Rodrigues I, Walter SD, Hanley J. Ferenczy A, et al., Canadian Cervical Cancer Screening Trial Study Group. Human papillomavirus DNA versus Papanicolaou screening tests for cervical cancer. N Eng/ J Med 2007; 357:1579-1588.

14. Naucler P, Ryd W, Tornberg S, Strand A, Wadell G, Elfgren K, et al. Human papillomavirus and Papanicolaou tests to screen for cervical cancer. N Engl J Med 2007; 357:1589-1597.

15. Kitchener HC, Almonte M, Thomson C, Wheeler P, Sargent A, Stoykova B, et al. HPV testing in combination with liquid-based cytology in primary cervical screening (ARTISTIC): a randomised controlled trial. Lancet Oncol 2009; 10:672-682.

16. Rijkaart DC, Berkhof J, Rozendaal L, van Kemenade FJ, Bulkmans NW, Heideman DA, et al. Human papillomavirus testing for the detection of high-grade cervical intraepithelial neoplasia and cancer: final results of the POBASCAM randomised controlled trial. Lancet Oncol 2012; 13:78-88.

17. Ronco G, Giorgi-Rossi P, Carozzi F, Confortini M, Dalla Palma P, Del Mistro A, et al., New Technologies for Cervical Cancer screening (NTCC) Working Group. Efficacy of human papillomavirus testing for the detection of invasive cervical cancers and cervical intraepithelial neoplasia: a randomised controlled trial. Lancet Oncol 2010; 11:249-257.

18. Anttila A, Kotaniemi-Talonen L, Leinonen $M$, Hakama $M$, Laurila P. Tarkkanen J, et al. Rate of cervical cancer, severe intraepithelial neoplasia, and adenocarcinoma in situ in primary HPV DNA screening with cytology triage: randomised study within organised screening programme. BMJ 2010; 340:c1804.

19. Ronco G, Dillner J, Elfstrom KM, Tunesi S, Snijders PJ, Arbyn M, et al., International HPV screening working group. Efficacy of HPV-based screening for prevention of invasive cervical cancer: follow-up of four European randomised controlled trials. Lancet 2014; 383:524-532.

20. Arbyn M, Ronco G, Anttila A, Meijer Cl, Poljak M, Ogilvie G, et al. Evidence regarding human papillomavirus testing in secondary prevention of cervical cancer. Vaccine 2012; 30 (Suppl 5):F88-F99.

21. Gage JC, Schiffman M, Katki HA, Castle PE, Fetterman B, Wentzensen $\mathrm{N}$, et al. Reassurance against future risk of precancer and cancer conferred by a negative human papillomavirus test. I Natl Cancer Inst 2014; 106:. 
22. Dijkstra MG, van Zummeren M, Rozendaal L, van Kemenade FJ, Helmerhorst TJ, Snijders PJ, et al. Safety of extending screening intervals beyond five years in cervical screening programmes with testing for high risk human papillomavirus: 14 year follow-up of population based randomised cohort in the Netherlands. $B M$ J 2016; 355:i4924.

23. Cuzick J, Clavel C, Petry KU, Meijer CJ, Hoyer H, Ratnam S, et al. Overview of the European and North American studies on HPV testing in primary cervical cancer screening. Int / Cancer 2006; 119:1095-1101.

24. Arbyn M, Sasieni P, Meijer CJ, Clavel C, Koliopoulos G, Dillner J. Chapter 9: clinical applications of HPV testing: a summary of meta-analyses. Vaccine 2006; 24 (Suppl 3):S3/78-S3/89.

25. Rijkaart DC, Berkhof J, van Kemenade FJ, Coupe VM, Hesselink AT, Rozendaal L, et al. Evaluation of 14 triage strategies for HPV DNA-positive women in population-based cervical screening. Int / Cancer 2012; 130:602-610.

26. Dijkstra MG, van Niekerk D, Rijkaart DC, van Kemenade FJ Heideman DA, Snijders PJ, et al. Primary hrHPV DNA testing in cervical cancer screening: how to manage screen-positive women? A POBASCAM trial substudy. Cancer Epidemiol Biomarkers Prev 2014; 23:55-63.

27. Steenbergen RD, Snijders PJ, Heideman DA, Meijer CJ. Clinical implications of (epi)genetic changes in HPV-induced cervical precancerous lesions. Nat Rev Cancer 2014; 14:395-405.

28. Luttmer R, De Strooper LM, Steenbergen RD, Berkhof I, Snijders PJ, Heideman DA, Meijer CJ. Management of high-risk HPVpositive women for detection of cervical (pre)cancer. Expert Rev Mol Diagn 2016; 16:961-974.

29. Wentzensen N, Sherman ME, Schiffman M, Wang SS. Utility of methylation markers in cervical cancer early detection: appraisal of the state-of-the-science. Gynecol Oncol 2009; 112:293-299.

30. Tornesello ML, Buonaguro L, Giorgi-Rossi P, Buonaguro FM Viral and cellular biomarkers in the diagnosis of cervical intraepithelial neoplasia and cancer. Biomed Res Int 2013; 2013:519619.

31. Steenbergen RD, Kramer D, Braakhuis BJ, Stern PL, Verheijen $\mathrm{RH}$, Meijer CJ, Snijders PJ. TSLC1 gene silencing in cervical cancer cell lines and cervical neoplasia. / Nat/ Cancer Inst 2004; 96:294-305.

32. Overmeer RM, Henken FE, Snijders PJ, Claassen-Kramer D, Berkhof J, Helmerhorst TJ, et al. Association between dense CADM1 promoter methylation and reduced protein expression in high-grade CIN and cervical SCC. J Pathol 2008; 215:388-397.

33. Overmeer RM, Henken FE, Bierkens M, Wilting SM, Timmerman I, Meijer CJ, et al. Repression of MAL tumour suppressor activity by promoter methylation during cervical carcinogenesis. I Pathol 2009; 219:327-336.

34. Wilting SM, van Boerdonk RA, Henken FE, Meijer CJ, Diosdado $B$, Meijer GA, et al. Methylation-mediated silencing and tumour suppressive function of hsa-miR-124 in cervical cancer. Mol Cancer 2010; 9:167.

35. Overmeer RM, Louwers JA, Meijer CJ, van Kemenade FJ, Hesselink AT, Daalmeijer NF, et al. Combined CADM1 and MAL promoter methylation analysis to detect (pre-)malignant cervical lesions in high-risk HPV-positive women. Int J Cancer 2011; 129:2218-2225.

36. Bierkens $M$, Hesselink AT, Meijer CJ, Heideman DA, Wisman GB, van der Zee AG, et al. CADM1 and MAL promoter methylation levels in hrHPV-positive cervical scrapes increase proportional to degree and duration of underlying cervical disease. Int I Cancer 2013; 133:1293-1299.

37. De Strooper LM, van Zummeren M, Steenbergen RD, Bleeker MC, Hesselink AT, Wisman GB, etal. CADM1, MAL and miR1242 methylation analysis in cervical scrapes to detect cervical and endometrial cancer. I Clin Pathol 2014; 67:1067-1071.

38. De Vuyst H, Franceschi S, Plummer M, Mugo NR, Sakr SR Meijer CJ, et al. Methylation levels of CADM1, MAL, and MIR124-2 in cervical scrapes for triage of HIV-infected, high-risk HPV-positive women in Kenya. I Acquir Immune Defic Syndr 2015; 70:311-318.

39. PreCursor-M kit Manufacturers' instructions 2015

40. Trienekens SCMKF, van den Broek IVF, Vriend HJ, Op de Coul ELM, van Veen MG, van Sighem Al, et al. Sexually transmitted infections, including HIV, in the Netherlands in 2011. Centre for Infectious Disease Control, National Institute for Public Health and the Environment 2012.
41. Solomon D, Davey D, Kurman R, Moriarty A, O'Connor D, Prey $M$, et al., Forum Group Members; Bethesda 2001 Workshop. The 2001 Bethesda System: terminology for reporting results of cervical cytology. JAMA 2002; 287:2114-2119.

42. Wright TW, Ronnett BM, Kurman RJ, Ferenczy A. Precancerous lesions of the cervix. In: Kurman RJ, Ellenson LH, Ronnett BM,editors. Blaustein's pathology of the female genital tract Philedelphia: Springer: 2011.pp 193-252.

43. Hesselink AT, Heideman DA, Steenbergen RD, Coupe VM, Overmeer RM, Rijkaart D, et al. Combined promoter methylation analysis of CADM1 and MAL: an objective triage tool for high-risk human papillomavirus DNA-positive women. Clin Cancer Res 2011; 17:2459-2465.

44. Snijders PJ, van den Brule AJ, Jacobs MV, Pol RP, Meijer CJ. HPV DNA detection and typing in cervical scrapes. Methods $\mathrm{Mol}$ Med 2005; 119:101-114.

45. van den Brule AJ, Pol R, Fransen-Daalmeijer N, Schouls LM, Meijer CJ, Snijders PJ. GP5+/6+ PCR followed by reverse line blot analysis enables rapid and high-throughput identification of human papillomavirus genotypes. / Clin Microbiol 2002; 40:779-787.

46. Schmittgen TD, Livak KJ. Analyzing real-time PCR data by the comparative C(T) method. Nat Protoc 2008; 3:1101-1108.

47. Pepe M. The statistical evaluation of medical tests for classification and prediction. Oxford: Oxford University Press; 2003.

48. Mikovits JA, Young HA, Vertino P, Issa JP, Pitha PM, TurcoskiCorrales $S$, et al. Infection with human immunodeficiency virus type 1 upregulates DNA methyltransferase, resulting in de novo methylation of the gamma interferon (IFN-gamma) promoter and subsequent downregulation of IFN-gamma production. Mol Cell Biol 1998; 18:5166-5177.

49. Zhang Y, Li SK, Yi Yang K, Liu M, Lee N, Tang X, et al. Whole genome methylation array reveals the down-regulation of IGFBP6 and SATB2 by HIV-1. Sci Rep 2015; 5:10806.

50. Verlaat W, Snijders PJ, Novianti PW, Wilting SM, De Strooper LM, Trooskens G, et al. Genome-wide DNA methylation profiling reveals methylation markers associated with 3q gain for detection of cervical precancer and cancer. Clin Cancer Res 2017.

51. Luttmer R, De Strooper LM, Berkhof J, Snijders PJ, Dijkstra MG, Uijterwaal $\mathrm{MH}$, et al. Comparing the performance of FAM19A4 methylation analysis, cytology and HPV16/18 genotyping for the detection of cervical (pre)cancer in high-risk HPV-positive women of a gynecologic outpatient population (COMETH study). Int / Cancer 2016; 138:992-1002.

52. Firnhaber C, Mayisela N, Mao L, Williams S, Swarts A, Faesen $M$, et al. Validation of cervical cancer screening methods in HIV positive women from Johannesburg South Africa. PLOS One 2013; 8:e53494.

53. Segondy M, Kelly H, Magooa MP, Djigma F, Ngou J, Gilham C, et al. Performance of careHPV for detecting high-grade cervical intraepithelial neoplasia among women living with HIV-1 in Burkina Faso and South Africa: HARP study. $\mathrm{Br} / \mathrm{Cancer}$ 2016; 115:425-430.

54. Nye MD, Hoyo C, Huang Z, Vidal AC, Wang F, Overcash F, et al. Associations between methylation of paternally expressed gene 3 (PEG3), cervical intraepithelial neoplasia and invasive cervical cancer. PLoS One 2013; 8:e56325.

55. Vidal AC, Henry NM, Murphy SK, Oneko O, Nye M, Bartlett JA, et al. PEG1/MEST and IGF2 DNA methylation in CIN and in cervical cancer. Clin Transl Oncol 2014; 16:266-272.

56. Luttmer R, Dijkstra MG, Snijders PJ, Berkhof J, van Kemenade FJ, Rozendaal L, et al. p16/Ki-67 dual-stained cytology for detecting cervical (pre)cancer in a HPV-positive gynecologic outpatient population. Mod Pathol 2016; 29:870-878.

57. Luttmer R, De Strooper LM, Dijkstra MG, Berkhof J, Snijders PJ, Steenbergen RD, et al. FAM19A4 methylation analysis in selfsamples compared with cervical scrapes for detecting cervical (pre)cancer in HPV-positive women. $\mathrm{Br} /$ Cancer 2016; 115:579-587.

58. Verhoef VM, Bosgraaf RP, van Kemenade FI, Rozendaal L, HeidemanDA, HesselinkAT, etal. Triage by methylation-marker testing versuscytology in women who test $\mathrm{HPV}$-positiveon self-collected cervicovaginal specimens (PROHTECT-3): a randomised controlled noninferiority trial. Lancet Oncol 2014; 15:315-322.

59. Bosgraaf RP, Siebers AG, De Hullu JA, Massuger LF, Bulten J, Bekkers RL, Melchers WJ. The current position and the future perspectives of cervical cancer screening. Expert Rev Anticancer Ther 2014; 14:75-92. 